Subscriber access provided by King Abdullah University of Science and Technology Library

Article

\title{
Substrate Lattice-Guided Seed Formation Controls the Orientation of 2D Transition Metal Dichalcogenides
}

Areej Aljarb, Zhen Cao, Hao-Ling Tang, Jing-Kai Huang, Mengliu Li, Weijin Hu, Luigi Cavallo, and Lain-Jong Li ACS Nano, Just Accepted Manuscript • DOI: 10.1021/acsnano.7b04323 • Publication Date (Web): 07 Aug 2017

Downloaded from http://pubs.acs.org on August 13, 2017

\section{Just Accepted}

"Just Accepted" manuscripts have been peer-reviewed and accepted for publication. They are posted online prior to technical editing, formatting for publication and author proofing. The American Chemical Society provides "Just Accepted" as a free service to the research community to expedite the dissemination of scientific material as soon as possible after acceptance. "Just Accepted" manuscripts appear in full in PDF format accompanied by an HTML abstract. "Just Accepted" manuscripts have been fully peer reviewed, but should not be considered the official version of record. They are accessible to all readers and citable by the Digital Object Identifier (DOI®). "Just Accepted" is an optional service offered to authors. Therefore, the "Just Accepted" Web site may not include all articles that will be published in the journal. After a manuscript is technically edited and formatted, it will be removed from the "Just Accepted" Web site and published as an ASAP article. Note that technical editing may introduce minor changes to the manuscript text and/or graphics which could affect content, and all legal disclaimers and ethical guidelines that apply to the journal pertain. ACS cannot be held responsible for errors or consequences arising from the use of information contained in these "Just Accepted" manuscripts. 


\section{The aligned growth of 2D monolayers is achieved with the controlled orientation of seeds, where a sulfur rich condition is required to minimize the size and define the orientation of seeds.}

$63 \times 39 \mathrm{~mm}(300 \times 300 \mathrm{DPI})$ 


\title{
Substrate Lattice-Guided Seed Formation Controls the Orientation of 2D Transition Metal Dichalcogenides
}

\author{
Areej Aljarb, Zhen Cao, Hao-Ling Tang, Jing-Kai Huang, Mengliu Li, Weijin Hu, Luigi Cavallo* \\ and Lain-Jong $L i^{\# *}$
}

King Abdullah University of Science and Technology, KAUST Catalysis Center, Physical Science and Engineering Division, Thuwal 23955-6900, Kingdom of Saudi Arabia.

*To whom correspondence should be addressed: luigi.cavallo@kaust.edu.sa or lance.li@kaust.edu.sa

\begin{abstract}
Two-dimensional (2D) transition metal dichalcogenide (TMDCs) semiconductors are important for next-generation electronics and optoelectronics. Given the difficulty in growing large single crystals of 2D TMDC materials, understanding the factors affecting the seed formation and orientation becomes an important issue for controlling the growth. Here, we systematically study the growth of molybdenum disulfide $\left(\mathrm{MoS}_{2}\right)$ monolayer on c-plane sapphire with chemical vapor deposition (CVD) to discover the factors controlling their orientation. We show that the concentration of precursors, i.e., the ratio between sulfur and molybdenum oxide $\left(\mathrm{MoO}_{3}\right)$, plays a key role in the size and orientation of seeds, subsequently controlling the orientation of $\mathrm{MoS}_{2}$ monolayers. High $\mathrm{S} / \mathrm{MoO}_{3}$ ratio is needed in the early stage of growth to form small seeds that can align easily to the substrate lattice structures while the ratio should be decreased to enlarge the size of the monolayer at the next stage of the lateral growth. Moreover, we show that the seeds are actually crystalline $\mathrm{MoS}_{2}$ layers as revealed by high-resolution transmission electron microscopy. There exist two preferred orientations $\left(0^{\circ}\right.$ or $\left.60^{\circ}\right)$ registered on sapphire, confirmed by our density functional theory (DFT) simulation. This report offers a facile technique to grow highly aligned 2D TMDCs and contributes to knowledge advancement in growth mechanism.
\end{abstract}


KEYWORDS: Two-dimensional materials; Molybdenum disulfide $\left(\mathrm{MoS}_{2}\right)$; Transition metal dichalcogenides; Chemical vapor deposition; Seeds; Aligned growth.

Two-dimensional (2D) transition metal dichalcogenides (TMDCs) have attracted significant attention owing to their unique electrical, ${ }^{1-2}$ optical, ${ }^{3-5}$ mechanical ${ }^{6}$ and thermal ${ }^{7}$ properties inherited from their 2D structures. In clear contrast to the semi-metallic graphene or the unstable black phosphorous, ${ }^{8}$ the ambient stable 2D TMDCs demonstrate promising properties for electronic and photoelectronic applications, such as field-effect transistors, ${ }^{1,9}$ sensors, ${ }^{10-11}$ solar cells ${ }^{12}$ and photodetectors. ${ }^{3,13-14}$ The molybdenum disulfide $\left(\mathrm{MoS}_{2}\right)$ monolayer, with a direct energy gap, ${ }^{15}$ strong photoluminescence, ${ }^{16}$ efficient valley and spin control, ${ }^{2}$ is one of the most intensively explored member in the 2D TMDCs family. It can be obtained by mechanical,${ }^{17}$ chemical ${ }^{18,19}$ or electrochemical ${ }^{20}$ exfoliation. However, these strategies lack uniformity and produce defect-rich samples, which may not be suitable for largescale device fabrication. For this reason, chemical vapor deposition (CVD) methods using the solid precursors $\mathrm{MoO}_{3}$ and $\mathrm{S}$ powders have been developed. ${ }^{21}$

Nevertheless, the developed CVD methods can not guarantee to synthesize defectless TMDCs with uniform thickness and orientation. The grain boundary between different domains can lower the quality of TMDCs as electronic devices by breaking the structural periodicity and introducing strain defects. Huang et al. have further shown that the electronic structures of the grain boundary strongly depends on the misorientation angle of adjacent domains. ${ }^{22}$ Hence, recent research efforts have dedicated special attention to control the orientation in order to obtain a large-scale and grain boundary-free monolayer films for higher electron mobility. ${ }^{23}$ Owing to the lattice symmetry of the 2D TMDCs layers, they are typically grown as triangles, hexagons or truncated triangles. ${ }^{24-25}$ The growth of aligned TMDCs triangles has been achieved on top of other crystalline layered materials such as graphite, ${ }^{26}$ graphene ${ }^{27-29}$ or boron nitrides ${ }^{30}$ where the TMDC monolayers are either aligned by the graphite step edges or with the substrate lattice through van der Waals (vdW) interaction. The aligned growth has also been 
successfully achieved on single crystal substrates with compatible lattice constants such as GaN, ${ }^{31-32}$ mica $^{33}$ and sapphire. ${ }^{34-35}$

Sapphire has been widely used as a substrate for the growth of 2D TMDC layers owing to its special lattice constant and flat insulating surfaces. Many reports have adopted c-plane sapphire as the substrate for growing TMDC layers. ${ }^{17,30,31}$ However, it is known that the sapphire surface can be reconstructed at high temperatures to form long terraces and wide steps. ${ }^{36}$ Chen, et al. have reported that the step-edges formed on the c-plane sapphire guided the aligned growth of $\mathrm{WSe}_{2}{ }^{35}$ where the alignment became prominent only at the temperatures as high as $950{ }^{\circ} \mathrm{C}$. Meanwhile, Dumcenco, et al. reported that the key to achieving orientation alignment is the atomically smooth sapphire surfaces, where the sapphire needed to be annealed at a temperature of $1000{ }^{\circ} \mathrm{C}$ in air for $1 \mathrm{~h}$ just before the growth process. ${ }^{34}$ However, several other reports did achieve the aligned TMDC triangles without sapphire pre-annealing at such a high temperature prior to the growth. ${ }^{37}$ Apparently, the fundamental details of aligned growth are still not yet fully understood. Since many parameters in CVD also affect the growth of TMDCs, such as precursor ratio, pressure, temperature, a systematic study to understand the mechanism of orientation alignment is required. In this study, we report that step-edge or terrace formation on sapphire is not the deterministic factor for the aligned growth of $\mathrm{MoS}_{2}$. The highly oriented $\mathrm{MoS}_{2}$ monolayers can be achieved at a much lower temperature $\left(750{ }^{\circ} \mathrm{C}\right)$. The higher ratio of sulfur to $\mathrm{MoO}_{3}$ at the initial nucleation step leads to a relatively smaller size of seeds spontaneously formed on sapphire. Consequently, these small seeds can easily rotate to the energetically favorable position, which is determined by the lattice structure of the substrate, leading to preferred orientation and alignment of TMDCs. By contrast, a lower sulfur to $\mathrm{MoO}_{3}$ ratio typically results in a larger size of seeds, which loses the capability of orientation control. High-resolution transmission electron microscopy (HRTEM) and energy dispersive X-ray analysis (EDX) reveal that the seeds are also crystalline $\mathrm{MoS}_{2}$ layers. Density functional theory (DFT) simulations confirm that the small $\mathrm{MoS}_{2}$ seeds exhibit preferred orientation on 
c-plane sapphire. This work provides a fundamental understanding of the seed formation and mechanism for orientation-controlled growth of TMDC monolayer.

\section{RESULTS AND DISCUSSION}

\section{Effect of $\mathrm{S} / \mathrm{MoO}_{3}$ precursor ratio on $\mathrm{MoS}_{2}$ alignment}

We adopt the CVD growth process first reported by Lee, et al. where the growth of $\mathrm{MoS}_{2}$ monolayer on sapphire relies on the gas phase reaction of $\mathrm{MoO}_{3}$ and sulfur vapors carried by a pure Ar flow. ${ }^{21}$ Sapphire is commonly used for $\mathrm{MoS}_{2}$ growth since it is an insulating substrate with high thermal stability and excellent crystalline quality. Additionally, sapphire and $\mathrm{MoS}_{2}$ both share a hexagonal crystal structure, which makes the growth of TMDCs on sapphire preferable. For this reason, the structure of the sapphire surface plays a key role in determining the $\mathrm{MoS}_{2}$ orientation through van der Waals (vdW) interaction. ${ }^{34}$ It is well known that various surface treatments on sapphire lead to different termination layers on its surfaces, e.g., $\mathrm{Al}, \mathrm{OH}$ or $\mathrm{O}$ termination, ${ }^{38}$ which may strongly affect the results of the $\mathrm{MoS}_{2}$ growth. For our $\mathrm{MoS}_{2}$ growth, sapphire is first treated with a piranha solution, resulting in $\mathrm{OH}$ group termination on the surfaces. Upon heating during the growth, two neighboring Al-OHs shall be dehydrated into $\mathrm{Al}_{2} \mathrm{O}_{3}{ }^{39}$ and thus the $\mathrm{MoS}_{2}$ is grown on the sapphire with an oxygen-terminated surface.

Figure 1a schematically illustrates the growth of $\mathrm{MoS}_{2}$ layers on c-plane sapphire substrates using the CVD process, and the heating profiles of the precursors sulfur and $\mathrm{MoO}_{3}$ powders are shown in Figure 1b. Other growth details are provided in the method section. In our typical CVD process, the sulfur powders (at heating zone 1) were first heated to $140{ }^{\circ} \mathrm{C}$ to fill up the reaction tube with $\mathrm{S}$ vapors, followed by the temperature ramping of $\mathrm{MoO}_{3}$ powders and sapphire substrates (at heating zone 2) to the growth temperature of $800{ }^{\circ} \mathrm{C}$ as illustrated in the heating profile $\mathrm{T}_{2 \mathrm{~A}}$ in Figure $1 \mathrm{~b}$. Interestingly, we observe drastically different growth behaviors at different locations of the same sapphire substrate. Figure 1c presents a schematic illustration of how the growth substrate is spatially separated into three 
locations. Figure $1 \mathrm{~d}$ shows the relative concentration of $\mathrm{MoO}_{3}$ at these locations based on the analysis of our separate experiment which determines the relative concentration of Mo deposits on substrates using EDX (details in supporting materials, Table S1 and Figure S1). The results clearly show that $\mathrm{MoO}_{3}$ vapor concentration fast decreases with the distance away from the $\mathrm{MoO}_{3}$ source. Note that in our experiments the sulfur vapor concentration is in excess relative to $\mathrm{MoO}_{3}$ inside the tube. Hence, our experiments were different from that described by Govind Rajan, et al., ${ }^{40}$ where $\mathrm{MoO}_{3}$ concentration was constant but $\mathrm{S}$ concentration decays along the reaction tube. In addition, the diffusivity of sulfur vapors is much higher than that of $\mathrm{MoO}_{3}{ }^{41}$ As a result, the $\mathrm{S} / \mathrm{MoO}_{3}$ ratio significantly increases with distance from the $\mathrm{MoO}_{3}$ source. The corresponding optical microscope (OM) images for the $\mathrm{MoS}_{2}$ monolayer flakes growing on these locations are shown in Figure 1e. In location I, the $\mathrm{MoS}_{2}$ flakes are randomly oriented but they start to show some alignment at location II. In location III, these $\mathrm{MoS}_{2}$ flakes exhibit dominant edge orientation $\left(0^{\circ}\right.$ and $60^{\circ}$ as detailed in the statistical analysis in supporting information Figure S2). The effect is not caused by the temperature variations since the temperatures at these locations are the same (within the range of $\pm 1{ }^{\circ} \mathrm{C}$ ). In previous reports, incrementing the $\mathrm{S} / \mathrm{MoO}_{3}$ ratio results in the geometry of the produced $\mathrm{MoS}_{2}$ shifting from hexagon to triangle. ${ }^{24-25,}{ }^{40}$ Our experiments always produce triangular $\mathrm{MoS}_{2}$ flakes, indicating the excess amount of sulfur vapor in the reaction tube. Besides, our experimental results clearly correlate the $\mathrm{S} / \mathrm{MoO}_{3}$ vapor ratio to the orientation control. We hypothesize that at high $\mathrm{S} / \mathrm{MoO}_{3}$ ratio the sulfur vapors efficiently reduce the $\mathrm{MoO}_{3}$ to form small $\mathrm{MoS}_{2}$ crystalline seeds, which have the ability to rotate and align with the lattice structure of the substrate. By contrast, low $\mathrm{S} / \mathrm{MoO}_{3}$ ratio may result in the incomplete sulfurization of $\mathrm{MoO}_{3}$, with relatively larger and thicker seeds. These sub-oxide nanoparticles may land on the sapphire substrate randomly. Consequently, the synthesized $\mathrm{MoS}_{2}$ flakes exhibit random orientations.

\section{Growth of aligned $\mathrm{MoS}_{2}$ monolayers with a seeding step}

Since a sulfur-rich environment is essential to form small $\mathrm{MoS}_{2}$ seeds as hypothesized above, the twostep heating profile $\mathrm{T}_{2 \mathrm{~B}}$ (shown in Figure $1 \mathrm{~b}$ ) is designed to achieve better alignment across the whole 
sample regions (including locations I, II and III), where the substrate first stay at $750{ }^{\circ} \mathrm{C}$ for $5 \mathrm{~min}$ before it is heated to the growth temperature. This step ensures a highly S-rich environment for completing the sulfurization of slightly evaporated $\mathrm{MoO}_{3}$ at the seed formation stage. Consequently, very small seeds can form and rotate easily to stay at a more energetically favorable orientation on the substrate. Although growing $\mathrm{MoS}_{2}$ flakes can be very slow at $750{ }^{\circ} \mathrm{C}$ due to insufficient $\mathrm{MoO}_{3}$ vapors, extending the growth time results in small monolayers with edge lengths less than $1 \mu \mathrm{m}$ as shown in Figure S3. This observation is consistent with Pan et al., where they revealed that at the low temperature of $750{ }^{\circ} \mathrm{C}$ tiny monolayers on Si substrate were obtained. ${ }^{42}$ Therefore, we designed a profile where after aligning small seeds at $750{ }^{\circ} \mathrm{C}$, the temperature is increased to $800{ }^{\circ} \mathrm{C}$ to increase the lateral growth and enlarge the monolayers size. Gratifyingly, we observed that all regions including I, II and III are grown with aligned $\mathrm{MoS}_{2}$ monolayers, see Figure 2. Figure 2a shows the typical OM images of the $\mathrm{MoS}_{2}$ monolayer flakes grown at the central region II using the heating profiles $\mathrm{T}_{2 \mathrm{~B}}$, where the $\mathrm{MoS}_{2}$ flakes are highly aligned and the dominant edge orientations are $0^{\circ}$ and $60^{\circ}$ as illustrated by the statistical analysis in Figure 2b. For comparison, one selected typical OM image for the $\mathrm{MoS}_{2}$ monolayers growth with the typical $\mathrm{T}_{2 \mathrm{~A}}$ profile (region II) is shown in Figure $2 \mathrm{c}$ and the corresponding statistical orientation analysis in Figure 2d clearly demonstrates the feature of random orientation. We have also performed separate experiments to examine the importance of the sulfur rich condition on $\mathrm{MoS}_{2}$ alignment by changing the feeding time of sulfur. The results in Figure S4 show that early feeding of S vapors results in better orientation alignment, strongly corroborating our hypothesis.

At a high temperature, c-plane sapphire would usually develop terrace structures with atomic steps on the surface. Aligned growth as guided by those steps has been reported for $\mathrm{WSe}_{2}$ on sapphire, where the growth temperature was above $950{ }^{\circ} \mathrm{C} .{ }^{35}$ However, in our case the growth temperature of $800{ }^{\circ} \mathrm{C}$ is not sufficiently high to conduct step edge-guided aligned growth. Consequently, our growth orientation is controlled by lattice crystal structure of the substrates instead of the terraces. Figure 2e shows an atomic 
force microscope (AFM) image for a monolayer $\mathrm{MoS}_{2}$ grown by our process on a sapphire substrate, where the edge of the flake is clearly not aligned with the steps from terrace structures.

\section{Structure of the seeds}

To explore the structure of the seeds, the growth is stopped after the seed formation stage $\left(750{ }^{\circ} \mathrm{C}\right.$ for $5 \mathrm{~min}$ in a sulfur-rich environment). AFM is adopted to characterize the morphology and size of the seeds initiated at this stage. Figure 3a is a typical AFM image for the seeds formed at the upstream side of the reaction zone. The shape of the seeds is identified as a triangle (more AFM images of the $\mathrm{MoS}_{2}$ seeds are provided in supporting information Figure S5). Unlike the results for one-step heating profile, the triangles in the upstream are aligned since the two-steps heating profile results in small seeds in all the substrate locations which can rotate and align to the substrate. Figure $3 \mathrm{~b}$ shows the cross-sectional height profile for the seed along the dashed line in Figure 3a, given a thickness of $\sim 0.6$ nm monolayer which agrees well with the reported thickness of $\mathrm{MoS}_{2}$ monolayers. ${ }^{43}$ Figure $3 \mathrm{c}$ displays the AFM image for the seeds formed at the downstream side with a thickness $\sim 1.2 \mathrm{~nm}$ (Figure $3 \mathrm{~d}$ ). The seeds at downstream side are normally with an apparent size of 20-30 nm. Note that the size may be overestimated since the lateral size measurement is limited by the size of the AFM tip end. Hence, identifying the shape of the seed is challenging. The size difference of the seeds in different regions may be related to the amount of the $\mathrm{MoO}_{3}$ vapor reaching the regions. It would require future investigations.

Most of the as-grown $\mathrm{MoS}_{2}$ monolayers do not exhibit identifiable nucleation center in the middle of the triangles under OM observation. However, we still occasionally find thick seeds with a pyramid shape as shown in Figure 4a. The cross-sectional HRTEM image in Figure $4 \mathrm{~b}$ confirms these thick seeds consist of stacked $\mathrm{MoS}_{2}$ layers, indicating even larger $\mathrm{MoO}_{3}$ can be completely sulfurized to form $\mathrm{MoS}_{2}$. The thickness is measured to be $7.5 \mathrm{~nm}$ corresponding to around 10 layers of $\mathrm{MoS}_{2}$. Additionally, Figure 4c shows the energy dispersive X-ray analysis (EDX) images for Al, O, Mo and S signals at the same location in Figure 4b. No $\mathrm{O}$ is observed in the $\mathrm{MoS}_{2}$ multilayers, which again confirms that the 
seed is composed of stacked $\mathrm{MoS}_{2}$ and the precursor $\mathrm{MoO}_{3}$ is completely sulfurized at the seed formation stage.

\section{Density functional theory simulation}

A more detailed investigation from the atomic viewpoint was performed through density functional theory (DFT) calculations. A model consisting of $4 \times 4$ unit cells of $\mathrm{Al}_{2} \mathrm{O}_{3}$ and $6 \times 6 \mathrm{MoS}_{2}$ was constructed to minimize the lattice constant mismatch to $0.7 \%$. As described, we believe the $\mathrm{MoS}_{2}$ monolayer is deposited on the sapphire c-plane with oxygen terminations, which is in clear contrast to the previously proposed models using the sapphire terminated with $\mathrm{H}$ or $\mathrm{Al}^{34}$ After optimization (Figure 5a), the vertical distance between the upper sulfur of $\mathrm{MoS}_{2}$ and the sapphire surface is $5.8 \AA$, which is comparable with our AFM characterization.

Since the seed only contains Mo and $\mathrm{S}$, it is represented by a more prototypical hexagon $\mathrm{MoS}_{2}$ piece as shown in Figure 5b. A systematic scanning consisting of over 7000 calculations with fixed relative orientations and positions between the $\mathrm{MoS}_{2}$ seed and $\mathrm{Al}_{2} \mathrm{O}_{3}$ substrate were performed, thereafter, to evaluate potential energy surface (PES) as a function of the relative position and orientation between the $\mathrm{MoS}_{2}$ seed and the sapphire substrate. Due to the heavy computational load, the distance between the top $\mathrm{O}$ layer of $\mathrm{Al}_{2} \mathrm{O}_{3}$ and the top $\mathrm{S}$ layer of $\mathrm{MoS}_{2}$ was fixed at $5.8 \AA$, which is the distance obtained from structural optimization. Figure $5 \mathrm{c}$ gives a description of the PES corresponding to an angle of $0^{\circ}$, and the other maps are shown in Figure S6. We have scanned large enough area to cover the $\mathrm{Al}_{2} \mathrm{O}_{3}$ surface region, and the distance between the maxima and minima of the PES is consistent with the distance between oxygen atoms at c-plane, indicating that the vdW interaction can tune the relative position of the $\mathrm{MoS}_{2}$ seeds landing on the surface. Thereafter, the configurations corresponding to the minimum in this PES is chosen for orientation scans of the $\mathrm{MoS}_{2}$ seed. For each specific orientation, the relative positions were also scanned to get a complete view of the PES. The representative configurations for each orientation were chosen from the minimum of the PES, and the energies were calculated with a higher precision (details in supporting materials). We obtain relative energy as a function of orientation 
angles with the most favorable position of the $\mathrm{MoS}_{2}$ seed as shown in Figure 5d. Considering the symmetry feature of our model, the results corresponding to $0 \sim 60$ degree relative orientation were demonstrated in this report.

Even though we simplify the simulation by using this small hexagon seed without considering the structural variation induced by a high experimental temperature, the simulation can give consistent trend compared with experiments: the $0^{\circ}$ or $60^{\circ}$ configurations are most favorable. It is also of interest to obtain further insights from this model. For instance, the PES is quite smooth and does not have large kinetic barriers ( $\sim 5 \mathrm{kBT}$ for experimental temperature), indicating the seed can smoothly find its energetic minimum. Consequently, in our experiment, a 5-minute plateau at $750{ }^{\circ} \mathrm{C}$ (Figure $1 \mathrm{~b}$ ) plays a role in assisting the small seeds finding their favorable configurations. Besides, any treatment destroying this smooth PES by generating additional large energy barriers may bring troubles by kinetically trapping the seed into a local minimum. In short, relatively flat substrate surface having periodic energetic minimums without significant energy barriers can be suitable to template the seed and generate highly aligned TMDC monolayers.

\section{CONCLUSION}

In conclusion, we have synthesized highly oriented $\mathrm{MoS}_{2}$ monolayers by controlling the sulfurization of $\mathrm{MoO}_{3}$ using $\mathrm{CVD}$ method. It is concluded that high sulfurization in the seed formation stage plays an important role to form a small seed, which can easily align with the lattice structure of the sapphire substrate. Seeds can be grown at $750{ }^{\circ} \mathrm{C}$, whereas higher temperature $800{ }^{\circ} \mathrm{C}$ speeds up the lateral growth. Additionally, we provide evidence that the seed is completely sulfurized and consists of $\mathrm{MoS}_{2}$. Such orientation control can be applicable for other 2D TMDC growth on a crystalline substrate. It is anticipated that such understanding of growth mechanism is crucially important for controlling the growth of diverse 2D materials and further understanding the aligning mechanism of TMDC growth on other hexagonal surfaces such as TMDC, BN, graphene and graphite heterostructure or single crystal substrates with compatible lattice constants such as GaN and mica. 


\section{MATERIALS AND METHODS}

Chemical Vapor Deposition of TMDCs. The $\mathrm{MoS}_{2}$ monolayer was grown by the chemical vapor deposition method. The precursors are $\mathrm{MoO}_{3}$ (Sigma-Aldrich, $\geq 99.5 \%$ purity) and $\mathrm{S}$ (Sigma-Aldrich, $\geq 99.5 \%$ purity) powders. The sulfur powder $(4 \mathrm{~g})$ was put at the upper stream side of the furnace (heating zone 1), and the temperature was maintained at $140{ }^{\circ} \mathrm{C}$ during the reaction. In the heating zone 2, center of the reaction chamber, the $\mathrm{MoO}_{3}$ powder $(0.8 \mathrm{~g})$ was placed in a ceramic boat with a $1 \mathrm{~cm} \times$ $5 \mathrm{~cm}$ sapphire substrate placed at the downstream of the ceramic boat. The gas flow was from $\mathrm{Ar}(\mathrm{Ar}=$ $90 \mathrm{sccm})$ and the chamber pressure was controlled at 40 Torr. First, the center of the furnace was gradually heated from room temperature to $750{ }^{\circ} \mathrm{C}$ at a ramping rate of $25{ }^{\circ} \mathrm{C} / \mathrm{min}$ and kept at this temperature for $5 \mathrm{~min}$. Then, the temperature was increased to the growth temperature $800{ }^{\circ} \mathrm{C}$ with the same ramping rate and kept for $10 \mathrm{~min}$. The furnace was then naturally cooled to room temperature. The consumption rate for $\mathrm{S}$ and $\mathrm{MoO}_{3}$ is estimated as $1.69 \mathrm{mg} / \mathrm{min}$ and $0.88 \mathrm{mg} / \mathrm{min}$ respectively (Sulfurrich reaction condition).

Characterization. Optical Images were collected using a Witec alpha 300 confocal Raman microscope with a RayShield coupler. The Mo concentration was analysed using scanning electron microscopy (SEM) imaging and energy dispersive X-ray (EDX) spectra which conducted using FEI Quanta 600 EDAX operating at $10 \mathrm{kV}$. The seeds and surface morphologies were examined on a commercial multifunction AFM instrument (Cypher ES model from Asylum Research Oxford Instruments) operating in contact mode. Olympus (OMCL-AC240TS) Al-coated silicon cantilevers were used for AFM characterizations. The resonance frequency was $\sim 70 \mathrm{kHz}$; the spring constant was $\sim 2 \mathrm{~N} / \mathrm{m}$, and the tip curvature radius was $\sim 7 \mathrm{~nm}$. The TEM cross-sectional samples were prepared in a Helios NanoLab 660 DualBeam FIB(focus ion Beam) system. Cross section HRTEM imaging and energy dispersive $\mathrm{X}$-ray (EDX) spectrum mapping data were conducted using FEI TITAN AND OSIRIS operating at $300 \mathrm{kV}$ and $200 \mathrm{kV}$ respectively. 
Density Functional Theory Modeling. The calculations started from the optimization of an $\mathrm{Al}_{2} \mathrm{O}_{3}$

slab consisting of 4 by 4 unit cells with 5 layers of oxides. The optimization were performed using the RPBE functional ${ }^{44}$ and the projected-augmented plane-wave method ${ }^{45}$ using the VASP package. ${ }^{46}$ The $^{4}$ vdW interactions were described through Grimme's correction. ${ }^{47}$ The energy cutoff was chosen as 400 $\mathrm{eV}$. The energy convergence criteria was chosen as $1.0 \times 10^{-6} \mathrm{eV}$, and the force convergence criteria was chosen as $1.0 \times 10^{-2} \mathrm{eV} / \AA$. Due to the computational load, the scanning process for the PES with different orientation angles uses $1 \times 1 \times 1 \mathrm{k}$ point (at the $\Gamma$ point), while the energy calculation for the chosen configurations uses $4 \times 4 \times 2 \mathrm{k}$ points using a Monkhorst-Pack grid. The scanning for Figure 5a is performed at a $0^{\circ}$ fixed angle over a $7 \times 7 \AA^{2}$ region with a $0.2 \AA$ grid length to cover larger area (longer than the terminal oxygen-oxygen distance). The scanning for other angles is to find the nearby energy minima, therefore, only cover a smaller $3 \times 3 \AA^{2}$ area with the $0.1 \AA$ grid length.

ACKNOWLEDGMENT All authors acknowledge support from King Abdullah University of Science and Technology (KAUST) under Competitive Research Grant (\#CRG4-2634) and KAUST Catalyst Center, Saudi Arabia. The simulations were performed on the Shaheen II supercomputer.

Supporting Information Available: Table S1 and Figures S1-S6 are included. This material is available free of charge via the Internet at http://pubs.acs.org.

\section{REFERENCES AND NOTES}

1. Radisavljevic, B.; Radenovic, A.; Brivio, J.; Giacometti, i. V.; Kis, A., Single-Layer $\mathrm{MoS}_{2}$ Transistors. Nat. Nanotechnol. 2011, 6, 147-150.

2. Zeng, H.; Dai, J.; Yao, W.; Xiao, D.; Cui, X., Valley Polarization in $\mathrm{MoS}_{2}$ Monolayers by Optical Pumping. Nat. Nanotechnol. 2012, 7, 490-493.

3. Lopez-Sanchez, O.; Lembke, D.; Kayci, M.; Radenovic, A.; Kis, A., Ultrasensitive Photodetectors Based on Monolayer MoS 2 . Nat. Nanotechnol. 2013, 8, 497-501.

4. Zhang, W.; Chuu, C.-P.; Huang, J.-K.; Chen, C.-H.; Tsai, M.-L.; Chang, Y.-H.; Liang, C.-T.; Chen, Y.-Z.; Chueh, Y.-L.; He, J.-H., et al., Ultrahigh-Gain Photodetectors Based on Atomically Thin Graphene- $\mathrm{MoS}_{2}$ Heterostructures. Sci. Rep. 2014, 4, 3826.

5. Cong, C.; Shang, J.; Wu, X.; Cao, B.; Peimyoo, N.; Qiu, C.; Sun, L.; Yu, T., Synthesis and Optical Properties of Large - Area Single - Crystalline 2D Semiconductor $\mathrm{WS}_{2}$ Monolayer from 
Chemical Vapor Deposition. Adv. Opt. Mater. 2014, 2, 131-136.

6. Bertolazzi, S.; Brivio, J.; Kis, A., Stretching and Breaking of Ultrathin $\mathrm{MoS}_{2}$. ACS Nano 2011, 5, 9703-9709.

7. Cai, Y.; Lan, J.; Zhang, G.; Zhang, Y.-W., Lattice Vibrational Modes and Phonon Thermal Conductivity of Monolayer MoS 2 . Phys. Rev. B 2014, 89, 035438.

8. Lin, S.; Chui, Y.; Li, Y.; Lau, S. P., Liquid-Phase Exfoliation of Black Phosphorus and Its Applications. FlatChem 2017, 2, 15-37.

9. Das, T.; Ahn, J.-H., Development of Electronic Devices Based on Two-Dimensional Materials. FlatChem 2017, 3, 43-63.

10. Perkins, F. K.; Friedman, A. L.; Cobas, E.; Campbell, P.; Jernigan, G.; Jonker, B. T., Chemical Vapor Sensing with Monolayer $\mathrm{MoS}_{2}$. Nano Lett. 2013, 13, 668-673.

11. Li, H.; Yin, Z.; He, Q.; Li, H.; Huang, X.; Lu, G.; Fam, D. W. H.; Tok, A. I. Y.; Zhang, Q.; Zhang, H., Fabrication of Single - and Multilayer $\mathrm{MoS}_{2}$ Film - Based Field - Effect Transistors for Sensing No at Room Temperature. Small 2012, 8, 63-67.

12. Van Le, Q.; Choi, J.-Y.; Kim, S. Y., Recent Advances in the Application of Two-Dimensional Materials as Charge Transport Layers in Organic and Perovskite Solar Cells. FlatChem 2017, 2, 54-66.

13. Zhang, W.; Huang, J. K.; Chen, C. H.; Chang, Y. H.; Cheng, Y. J.; Li, L. J., High-Gain Phototransistors Based on a CVD $\mathrm{MoS}_{2}$ Monolayer. Adv. Mater. 2013, 25, 3456-3461.

14. Yin, Z.; Li, H.; Li, H.; Jiang, L.; Shi, Y.; Sun, Y.; Lu, G.; Zhang, Q.; Chen, X.; Zhang, H., Single-Layer $\mathrm{MoS}_{2}$ Phototransistors. ACS Nano 2011, 6, 74-80.

15. Mak, K. F.; Lee, C.; Hone, J.; Shan, J.; Heinz, T. F., Atomically Thin $\mathrm{MoS}_{2}$ : A New Direct-Gap Semiconductor. Phys. Rev. Lett. 2010, 105, 136805.

16. Splendiani, A.; Sun, L.; Zhang, Y.; Li, T.; Kim, J.; Chim, C. Y.; Galli, G.; Wang, F., Emerging Photoluminescence in Monolayer $\mathrm{MoS}_{2}$. Nano Lett. 2010, 10, 1271-1275.

17. Li, H.; Wu, J.; Yin, Z.; Zhang, H., Preparation and Applications of Mechanically Exfoliated Single-Layer and Multilayer $\mathrm{MoS}_{2}$ and $\mathrm{WSe}_{2}$ Nanosheets. Acc. Chem. Res. 2014, 47, 1067-1075.

18. Eda, G.; Yamaguchi, H.; Voiry, D.; Fujita, T.; Chen, M.; Chhowalla, M., Photoluminescence from Chemically Exfoliated $\mathrm{MoS}_{2}$. Nano Lett. 2011, 11, 5111-5116.

19. Ortiz-Quiles, E. O.; Cabrera, C. R., Exfoliated Molybdenum Disulfide for Dye Sensitized Solar Cells. FlatChem 2017, 2, 1-7.

20. Zeng, Z.; Yin, Z.; Huang, X.; Li, H.; He, Q.; Lu, G.; Boey, F.; Zhang, H., Single-Layer Semiconducting Nanosheets: High - Yield Preparation and Device Fabrication. Angew. Chem., Int. Ed 2011, 50, 11093-11097.

21. Lee, Y. H.; Zhang, X. Q.; Zhang, W.; Chang, M. T.; Lin, C. T.; Chang, K. D.; Yu, Y. C.; Wang, J. T. W.; Chang, C. S.; Li, L. J., Synthesis of Large-Area $\mathrm{MoS}_{2}$ Atomic Layers with Chemical Vapor Deposition. Adv. Mater. 2012, 24, 2320-2325. 
22. Huang, Y. L.; Chen, Y.; Zhang, W.; Quek, S. Y.; Chen, C.-H.; Li, L.-J.; Hsu, W.-T.; Chang, W.H.; Zheng, Y. J.; Chen, W., Bandgap Tunability at Single-Layer Molybdenum Disulphide Grain Boundaries. Nat. Commun. 2015, 6, 6298.

23. Najmaei, S.; Amani, M.; Chin, M. L.; Liu, Z.; Birdwell, A. G.; O’Regan, T. P.; Ajayan, P. M.; Dubey, M.; Lou, J., Electrical Transport Properties of Polycrystalline Monolayer Molybdenum Disulfide. ACS Nano 2014, 8, 7930-7937.

24. Yang, S. Y.; Shim, G. W.; Seo, S.-B.; Choi, S.-Y., Effective Shape-Controlled Growth of Monolayer $\mathrm{MoS}_{2}$ Flakes by Powder-Based Chemical Vapor Deposition. Nano Res. 2017, 10, 255-262.

25. Wang, S.; Rong, Y.; Fan, Y.; Pacios, M.; Bhaskaran, H.; He, K.; Warner, J. H., Shape Evolution of Monolayer $\mathrm{MoS}_{2}$ Crystals Grown by Chemical Vapor Deposition. Chem. Mater. 2014, 26, 63716379.

26. Lu, C.-I.; Butler, C. J.; Huang, J.-K.; Hsing, C.-R.; Yang, H.-H.; Chu, Y.-H.; Luo, C.-H.; Sun, Y.-C.; Hsu, S.-H.; Yang, K.-H. O., Graphite Edge Controlled Registration of Monolayer $\mathrm{MoS}_{2}$ Crystal Orientation. Appl. Phys. Lett. 2015, 106, 181904.

27. Lin, Y.-C.; Lu, N.; Perea-Lopez, N.; Li, J.; Lin, Z.; Peng, X.; Lee, C. H.; Sun, C.; Calderin, L.; Browning, P. N., Direct Synthesis of Van Der Waals Solids. ACS Nano 2014, 8, 3715-3723.

28. Ago, H.; Endo, H.; Solís-Fernández, P.; Takizawa, R.; Ohta, Y.; Fujita, Y.; Yamamoto, K.; Tsuji, M., Controlled Van Der Waals Epitaxy of Monolayer $\mathrm{MoS}_{2}$ Triangular Domains on Graphene. ACS Appl. Mater. Interfaces 2015, 7, 5265-5273.

29. Ago, H.; Fukamachi, S.; Endo, H.; Solís-Fernández, P.; Mohamad Yunus, R.; Uchida, Y.; Panchal, V.; Kazakova, O.; Tsuji, M., Visualization of Grain Structure and Boundaries of Polycrystalline Graphene and Two-Dimensional Materials by Epitaxial Growth of Transition Metal Dichalcogenides. ACS Nano 2016, 10, 3233-3240.

30. Yu, H.; Yang, Z.; Du, L.; Zhang, J.; Shi, J.; Chen, W.; Chen, P.; Liao, M.; Zhao, J.; Meng, J., Precisely Aligned Monolayer $\mathrm{MoS}_{2}$ Epitaxially Grown on $\mathrm{H}$ - Bn Basal Plane. Small 2016, 13, 1603005.

31. Ruzmetov, D.; Zhang, K.; Stan, G.; Kalanyan, B.; Bhimanapati, G. R.; Eichfeld, S. M.; Burke, R. A.; Shah, P. B.; O'Regan, T. P.; Crowne, F. J., et al., Vertical 2d/3d Semiconductor Heterostructures Based on Epitaxial Molybdenum Disulfide and Gallium Nitride. ACS Nano 2016, 10, 3580-3588.

32. Chen, Z.; Liu, H.; Chen, X.; Chu, G.; Chu, S.; Zhang, H., Wafer-Size and Single-Crystal MoSe 2 Atomically Thin Films Grown on Gan Substrate for Light Emission and Harvesting. ACS Appl. Mater. Interfaces 2016, 8, 20267-20273.

33. Ji, Q.; Zhang, Y.; Gao, T.; Zhang, Y.; Ma, D.; Liu, M.; Chen, Y.; Qiao, X.; Tan, P.-H.; Kan, M., Epitaxial Monolayer $\mathrm{MoS}_{2}$ on Mica with Novel Photoluminescence. Nano Lett. 2013, 13, 3870-3877.

34. Dumcenco, D.; Ovchinnikov, D.; Marinov, K.; Lazic, P.; Gibertini, M.; Marzari, N.; Sanchez, O. L.; Kung, Y.-C.; Krasnozhon, D.; Chen, M.-W., Large-Area Epitaxial Monolayer $\mathrm{MoS}_{2}$. ACS Nano 2015, 9, 4611-4620.

35. Chen, L.; Liu, B.; Ge, M.; Ma, Y.; Abbas, A. N.; Zhou, C., Step-Edge-Guided Nucleation and 
Growth of Aligned $\mathrm{WSe}_{2}$ on Sapphire via a Layer-over-Layer Growth Mode. ACS Nano 2015, 9, 83688375.

36. Curiotto, S.; Chatain, D., Surface Morphology and Composition of C-, a-and M-Sapphire Surfaces in $\mathrm{O}_{2}$ and $\mathrm{H}_{2}$ Environments. Surf. Sci. 2009, 603, 2688-2697.

37. Huang, J.-K.; Pu, J.; Hsu, C.-L.; Chiu, M.-H.; Juang, Z.-Y.; Chang, Y.-H.; Chang, W.-H.; Iwasa,

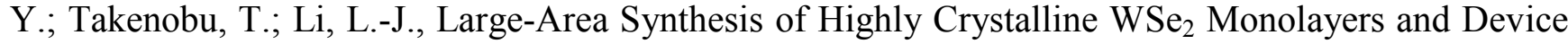
Applications. ACS Nano 2013, 8, 923-930.

38. Walters, C.; McCarty, K.; Soares, E.; Van Hove, M., The Surface Structure of $\alpha-\mathrm{Al}_{2} \mathrm{O}_{3}$ Determined by Low-Energy Electron Diffraction: Aluminum Termination and Evidence for Anomolously Large Thermal Vibrations. Surf. Sci. 2000, 464, L732-L738.

39. Živković, Ž. D.; Dobovišek, B., Kinetics of Aluminium Hydroxide Dehydration. J. Therm. Anal. Calorim. 1977, 12, 207-215.

40. Govind Rajan, A.; Warner, J. H.; Blankschtein, D.; Strano, M. S., Generalized Mechanistic Model for the Chemical Vapor Deposition of 2D Transition Metal Dichalcogenide Monolayers. ACS Nano 2016, 10, 4330-4344.

41. Shang, S.-L.; Lindwall, G.; Wang, Y.; Redwing, J. M.; Anderson, T.; Liu, Z.-K., Lateral Versus Vertical Growth of Two-Dimensional Layered Transition-Metal Dichalcogenides: Thermodynamic Insight into $\mathrm{MoS}_{2}$. Nano Lett. 2016, 16, 5742-5750.

42. Yang, X.; Li, Q.; Hu, G.; Wang, Z.; Yang, Z.; Liu, X.; Dong, M.; Pan, C., Controlled Synthesis of High-Quality Crystals of Monolayer $\mathrm{MoS}_{2}$ for Nanoelectronic Device Application. Sci. China Mater 2016, 59, 182-190.

43. Wieting, T.; Verble, J., Infrared and Raman Studies of Long-Wavelength Optical Phonons in Hexagonal MoS 2 . Phys. Rev. B 1971, 3, 4286.

44. Zhang, Y.; Yang, W., Comment on "Generalized Gradient Approximation Made Simple". Phys. Rev. Lett. 1998, 80, 890.

45. Blöchl, P. E., Projector Augmented-Wave Method. Phys. Rev. B 1994, 50, 17953.

46. Kresse, G.; Joubert, D., From Ultrasoft Pseudopotentials to the Projector Augmented-Wave Method. Phys. Rev. B 1999, 59, 1758.

47. Grimme, S.; Antony, J.; Ehrlich, S.; Krieg, H., A Consistent and Accurate Ab Initio Parametrization of Density Functional Dispersion Correction (DFT-D) for the 94 Elements. H-Pu. $J$. Chem. Phys. 2010, 132, 154104. 
(a)

Growth substrate

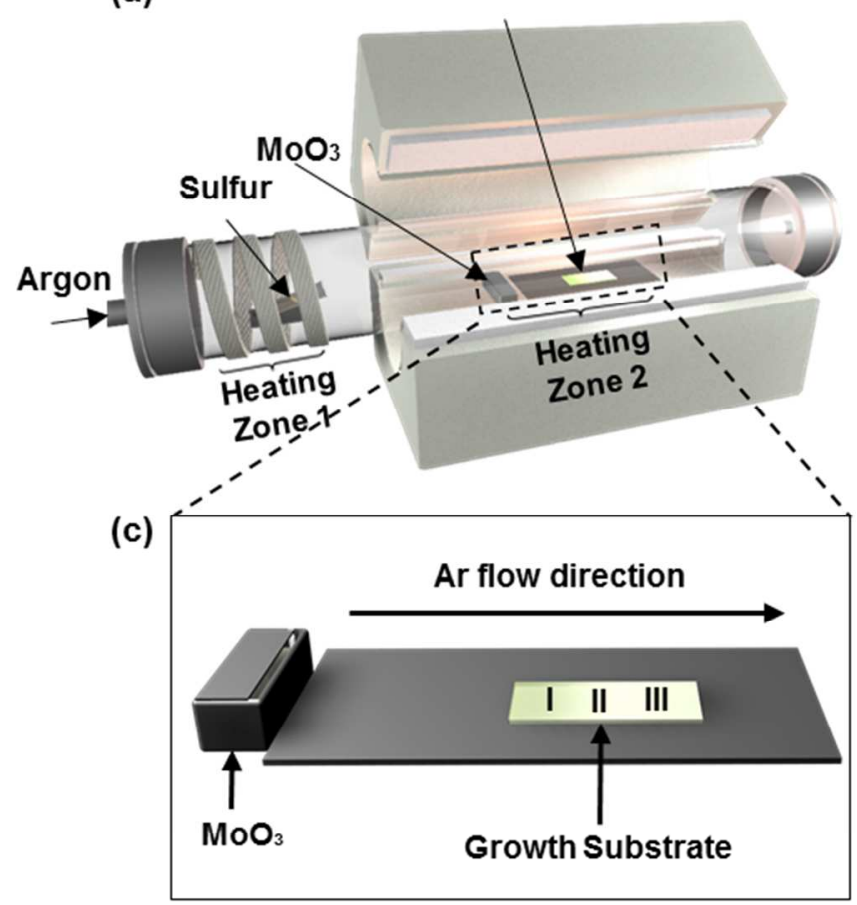

(b)

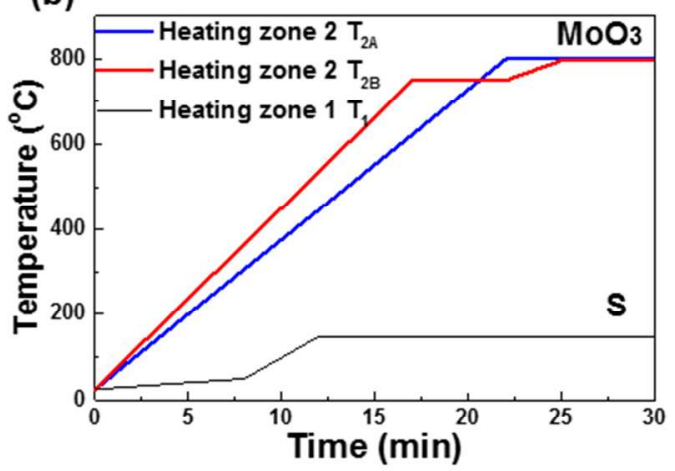

(d)

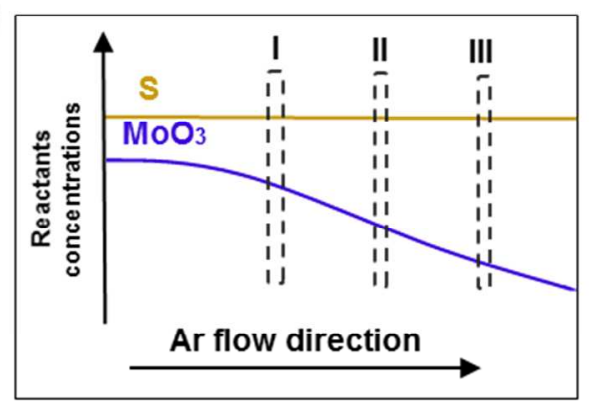

(e)

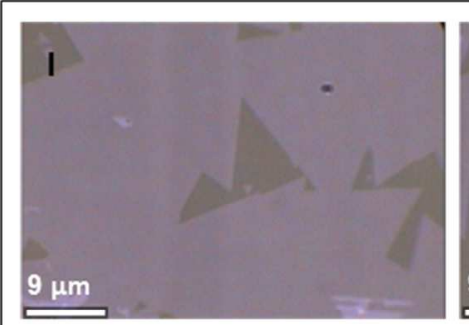

Low

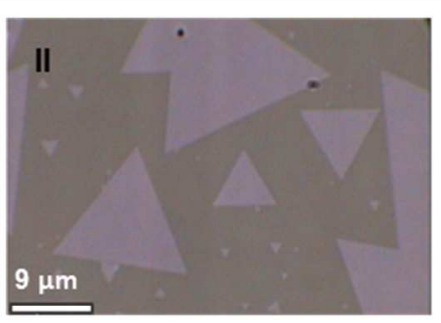

Alignment

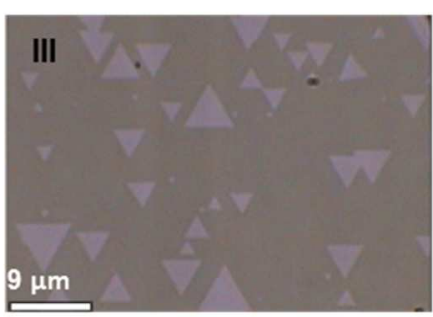

High

Figure 1. (a) Schematic illustration of the experimental setup of the $\mathrm{MoS}_{2}$ growth. (b) The temperature heating profiles adopted for the study of growing $\mathrm{MoS}_{2}$. (c) Schematic illustration for the various locations (I, II and III) according to the distance away from the $\mathrm{MoO}_{3}$ source. (d) A schematic illustration of the reactants concentration of $\mathrm{MoO}_{3}$ and $\mathrm{S}$ reached the specified locations in (c). (e)The corresponding $\mathrm{OM}$ images of the $\mathrm{MoS}_{2}$ flakes at each location. Scale bars: $9 \mu \mathrm{m}$. 

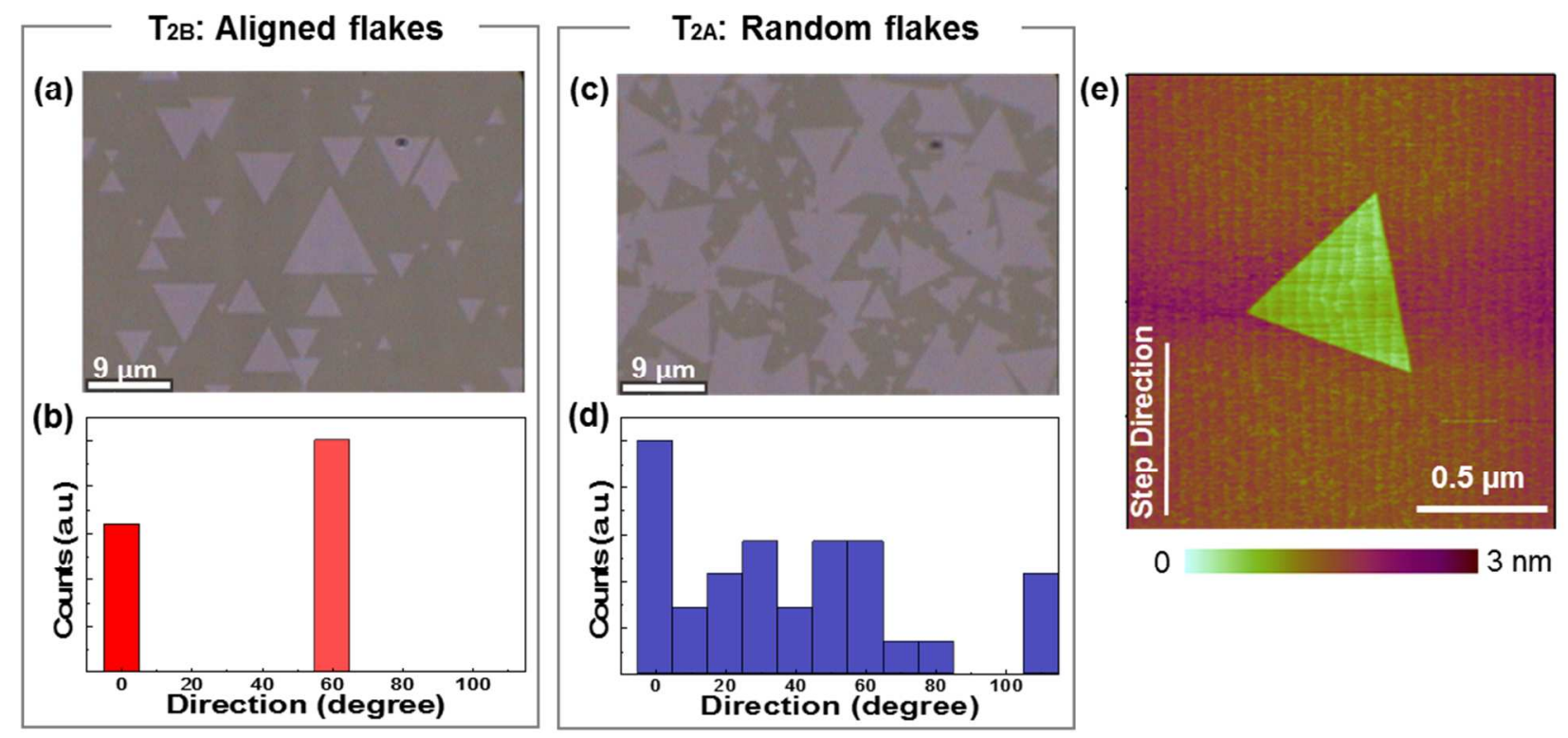

Figure 2. (a) The $\mathrm{OM}$ image of the as-grown $\mathrm{MoS}_{2}$ flakes using $\mathrm{T}_{2 \mathrm{~B}}$ heating profile. (b) Histograms of the orientation distributions based on the image (a). (c) The OM image of as-grown $\mathrm{MoS}_{2}$ flakes using the $\mathrm{T}_{2 \mathrm{~A}}$ heating profile. (d) Histograms of the orientation distributions based on the image (c). (e) AFM image of $\mathrm{MoS}_{2}$ monolayer flakes grown on c-plane sapphire using $\mathrm{T}_{2 \mathrm{~B}}$ heating profile. 
(a)

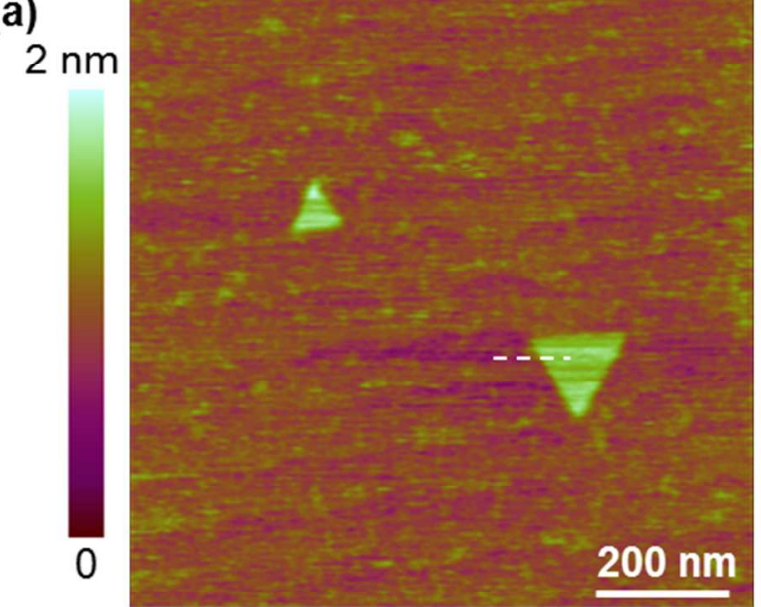

(c)

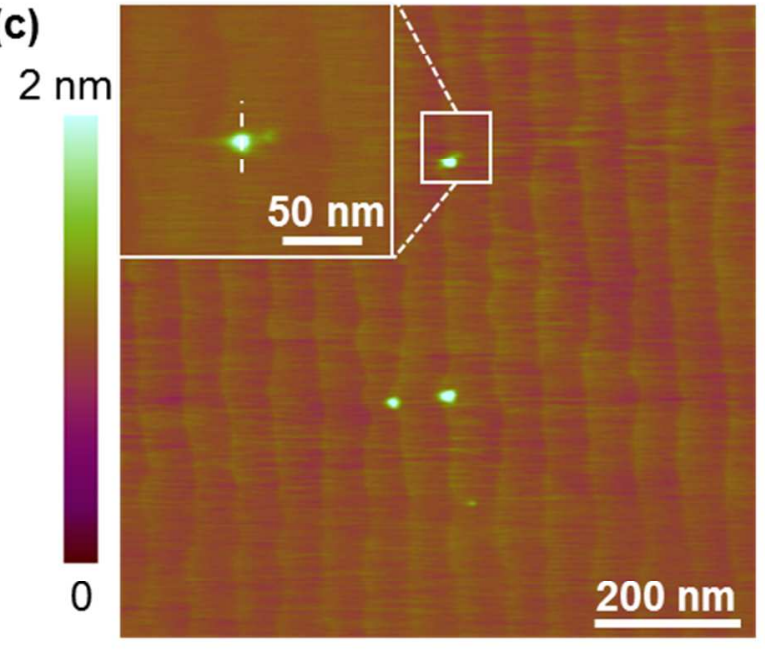

(b)

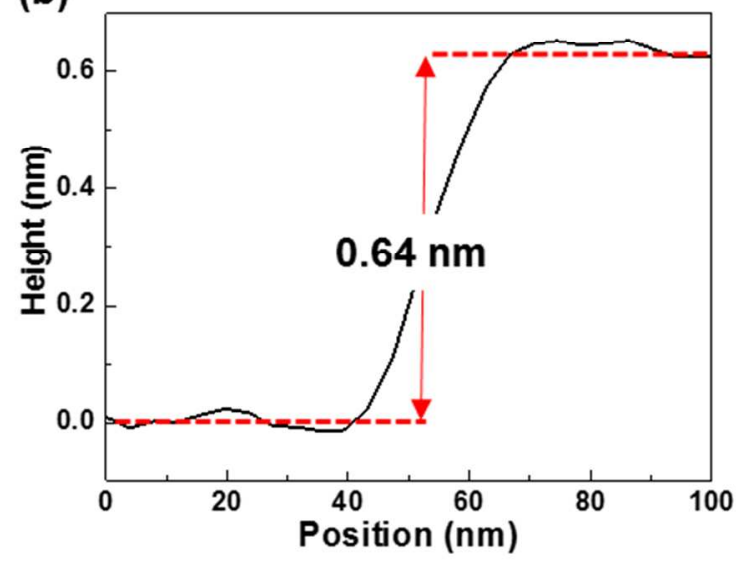

(d)

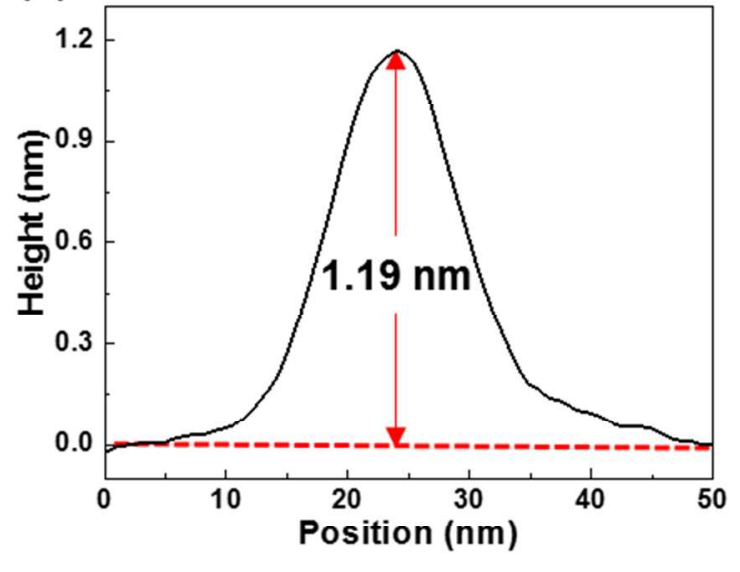

Figure 3. (a) AFM image of the $\mathrm{MoS}_{2}$ seeds at the upstream region. (b) Height profile for the white dashed line in (a). (c) AFM image of the $\mathrm{MoS}_{2}$ seeds at the downstream region. The inset in (c) shows zoomed AFM image for the white box. (d) Height profile for the white dashed line (c). 
(a)

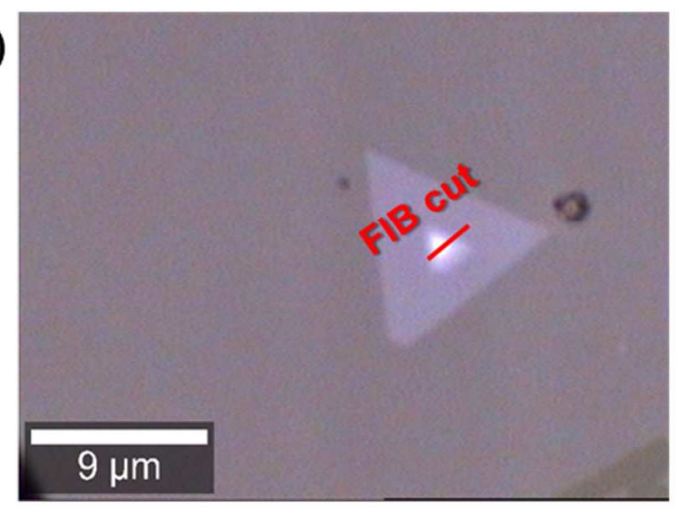

(c)
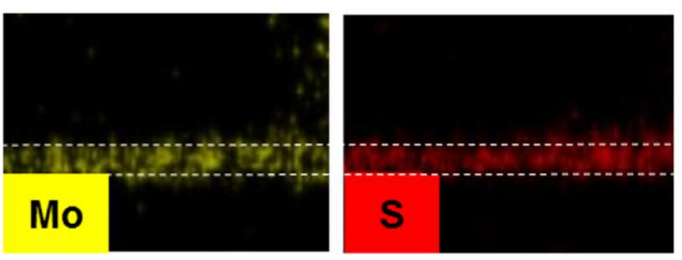

(b)
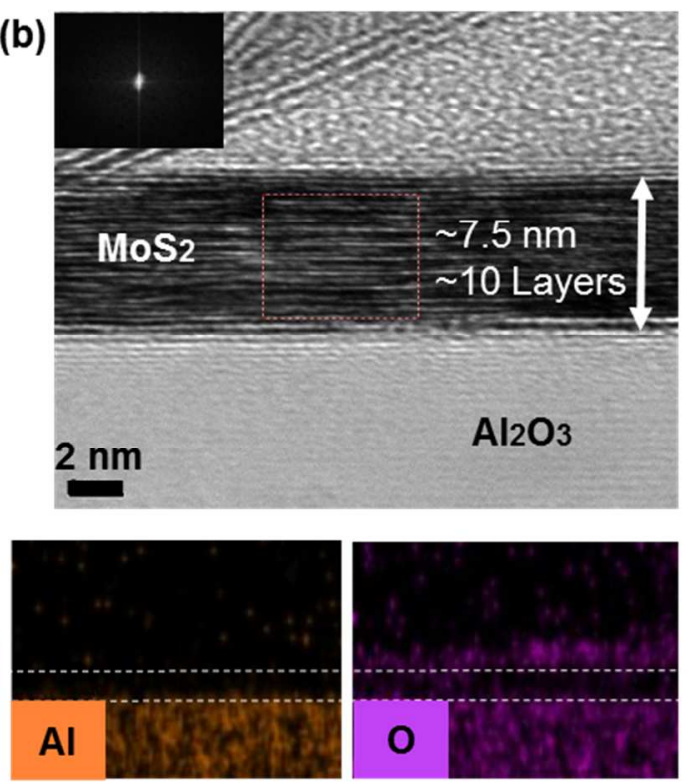

Figure 4. Cross-sectional HRTEM of $\mathrm{MoS}_{2}$ on c-plane sapphire demonstrating the seed composition. (a) The $\mathrm{OM}$ image of as-grown $\mathrm{MoS}_{2}$ flakes with a thick seed. The red line marks the area from which the FIB was cut; (b) Cross-sectional HRTEM image of $\mathrm{MoS}_{2}$ and (c) the corresponding EDX maps of (b) for Mo, S, Al and $\mathrm{O}$ signals. 
(a)

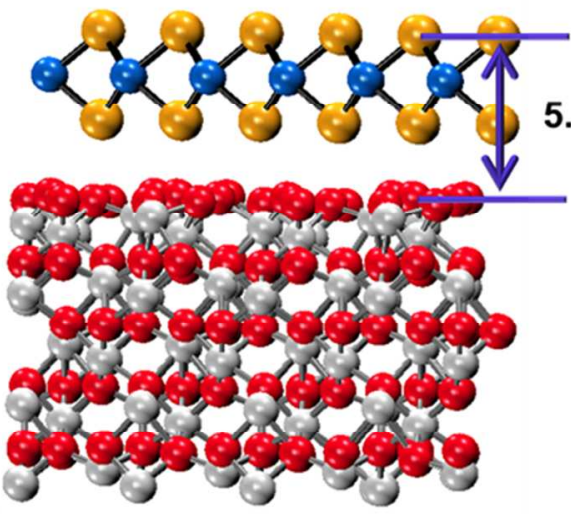

(c)

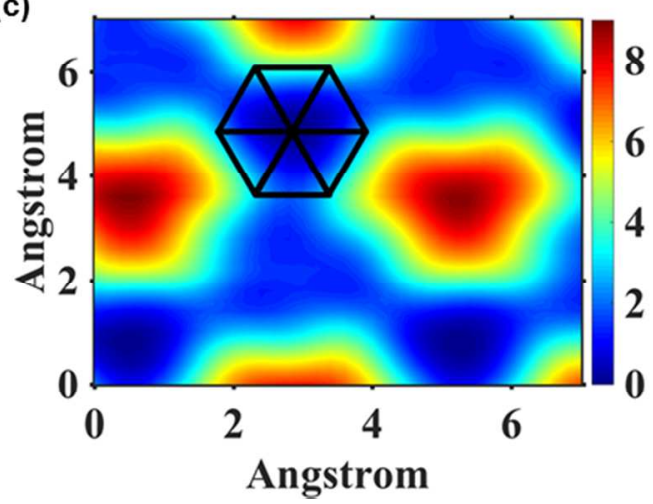

(b)

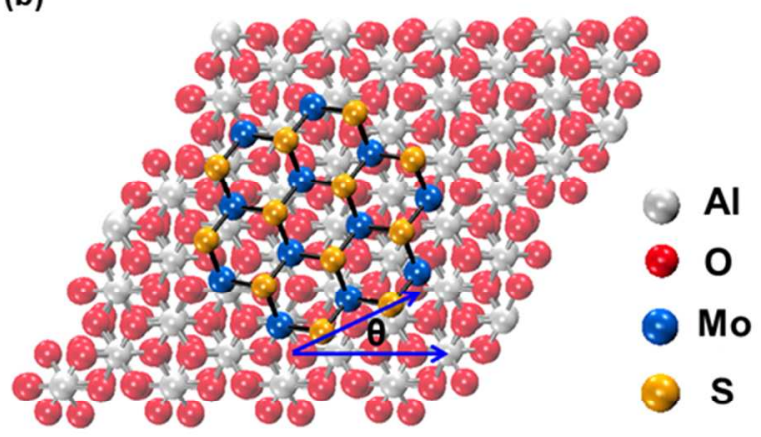

(d)

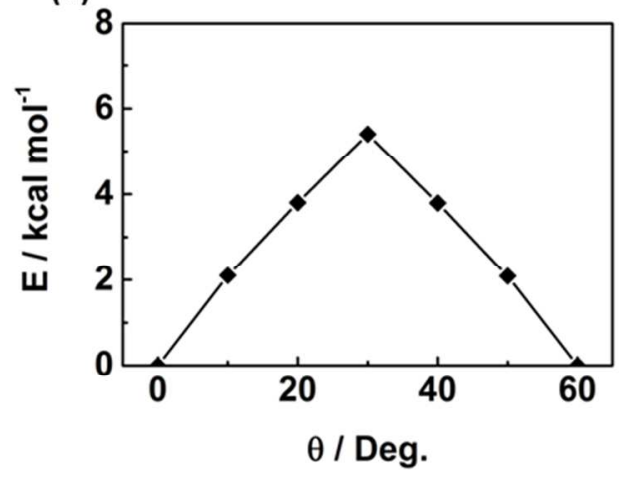

Figure 5. DFT calculation of the relative energy as a function between different orientation angles between $\mathrm{MoS}_{2}$ seed and sapphire substrate. (a) The optimized $\mathrm{MoS}_{2}$ layer on sapphire substrate demonstrates. (b) Schematic view of the $\mathrm{MoS}_{2}$ hexagon seed on the sapphire substrate. (c) Potential energy surface as a function of different relative position of $\mathrm{MoS}_{2}$ seed on sapphire substrate when the relative angle is fixed as 0 . (d) Potential energy as a function of relative angle between the $\mathrm{MoS}_{2}$ seed on sapphire; each configuration was chosen as the minimum point on the position scan $(0$ degree in $\mathrm{c}$, the rest in supporting information). 